\title{
Shallow Water Waves on a Deep Technology Stack: Accelerating a Finite Volume Tsunami Model using Reconfigurable Hardware in Invasive Computing
}

Alexander Pöppl1', Marvin Damschen², Florian Schmaus ${ }^{3}$, Andreas Fried², Manuel Mohr², Matthias Blankertz², Lars Bauer², Jörg Henkel², Wolfgang Schröder-Preikschat ${ }^{3}$, Michael Bader ${ }^{1}$

1) Technical University of Munich, Germany

2) Karlsruhe Institute of Technology, Germany

3) Friedrich-Alexander University Erlangen-Nürnberg, Germany

The 10th Workshop on UnConventional High Performance Computing 2017 August 28th, Santiago de Compostela, Spain 


\section{Motivation}

- Heterogeneous Environments commonplace in HPC

- NVidia Tesla GPUs, Intel Xeon Phi, ...

- New: Application-specific hardware (Google Tensor Processing Units, Microsoft Catapult, Anton 2)

- Reconfigurable fabric commonly used in embedded scenarios

- Performance comparable to ASICs

- May be reconfigured at run time.

- Special case: Reconfigurable fabric and CPU on the same chip

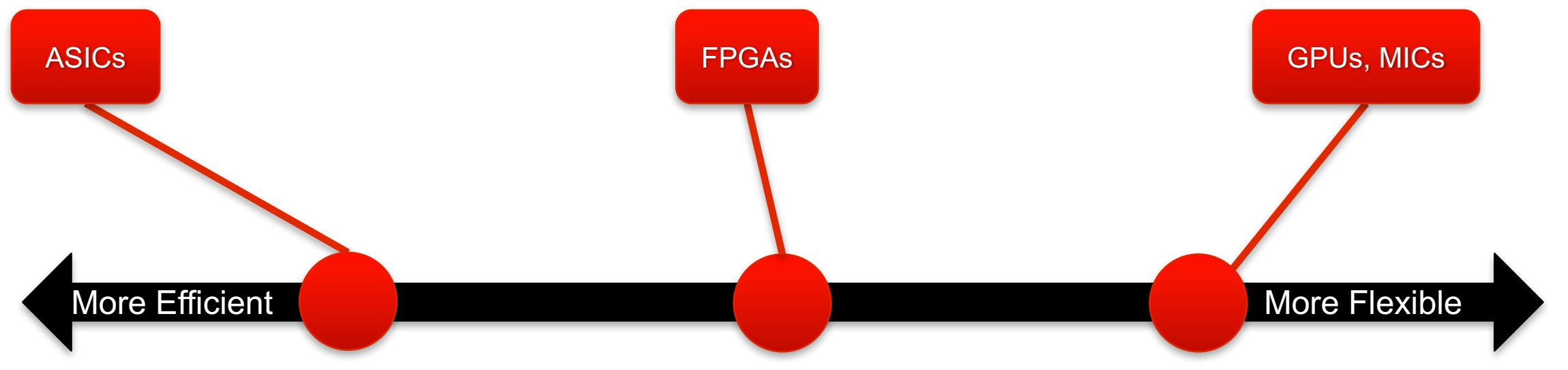


- Heterogeneous Environments commonplace in HPC

- NVidia Tesla GPUs, Intel Xeon Phi, ...

- New: Application-specific hardware (Google Tensor Processing Units, Microsoft Catapult, Anton 2)

- Reconfigurable fabric commonly used in embedded scenarios

- Performance comparable to ASICs

- May be reconfigured at run time.

- Special case: Reconfigurable fabric and CPU on the same chip






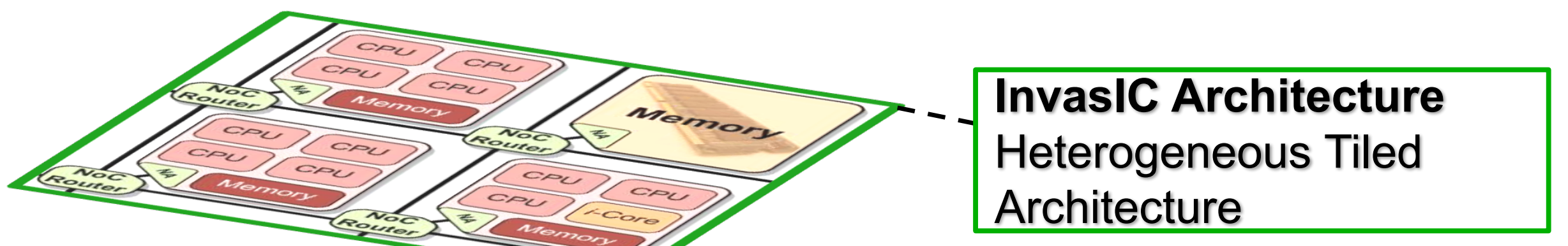









\section{Invasive Computing}
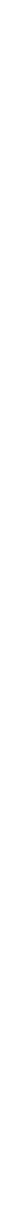

InvasIC Architecture Heterogeneous Tiled Architecture 
- Heterogeneous Multiprocessor System-on-Chip

- Tiled Architecture

- RISC Tiles

- $i$-Core Tiles

- Memory \& I/O Tiles

- No inter-tile cache coherence

- Connected through Network-on-Chip

- Heterogeneous Memory

- Tile-local Memory

- Global memory (Off-Tile, via NoC)






\section{OctoPOS - The Invasive Operating System}

- Parallel Operating System tailored for systems with $1000+$ cores

- Non-traditional threading scheme: $i$-lets

- Run-to-Completion semantics with cooperative scheduling

- Exclusive resource access

- Binding of $i$-let to execution context only at blocking operations

- Recycling of execution contexts:

Application



Free Contexts

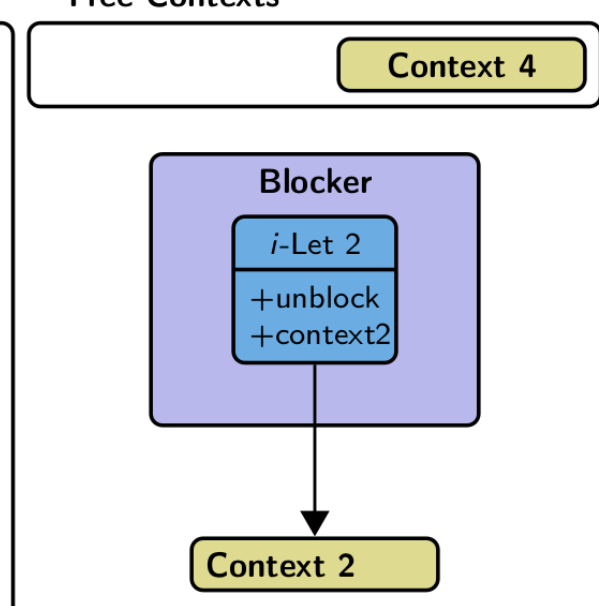
Little Overhead for creation, scheduling and dispatch 


\section{InvadeX10 - The Invasive Language}

- Asynchronous Partitioned Global Address Space (APGAS)

- Activities within an X10 Place may freely access objects allocated by activities spawned in the same Place

- Global Reference to objects in other places possible

- Remote objects not accessed directly, instead creation of copies or place-shift

- Natural fit for InvasIC

- Activities > $i$-lets

- Places > Tiles

- Serialization > Direct Cloning

- Invasive Compiler x10i

- Implements Resource-awareness (invade, infect, retreat)

- Direct use of OctoPOS APIs

- Emits Assembly (SPARC, x86)




- Proxy Application for simulation of shallow water waves

- Compute propagation of tsunamis given initial displacement

- Simulate inundation of coastal areas

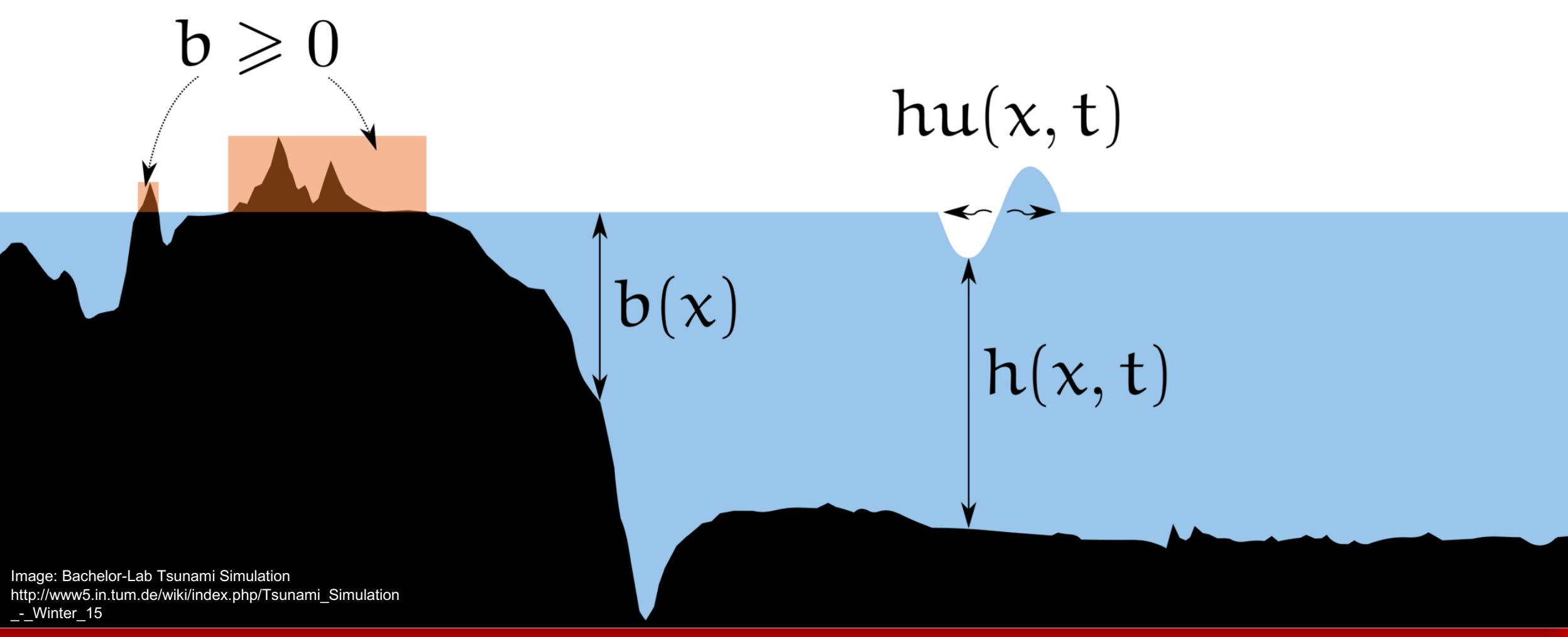


- Finite volume scheme on a Cartesian grid with piecewise constant unknown quantities and Eulerian time step

$$
\begin{aligned}
Q_{i, j}^{n+1}=Q_{i, j}^{n} & -\frac{\Delta t}{\Delta x}\left(\mathcal{A}^{+} \Delta Q_{i-\frac{1}{2}, j}+\mathcal{A}^{-} \Delta Q_{i+\frac{1}{2}, j}^{n}\right) \\
& -\frac{\Delta t}{\Delta y}\left(\mathcal{B}^{+} \Delta Q_{i, j-\frac{1}{2}}+\mathcal{B}^{-} \Delta Q_{i, j+\frac{1}{2}}^{n}\right)
\end{aligned}
$$

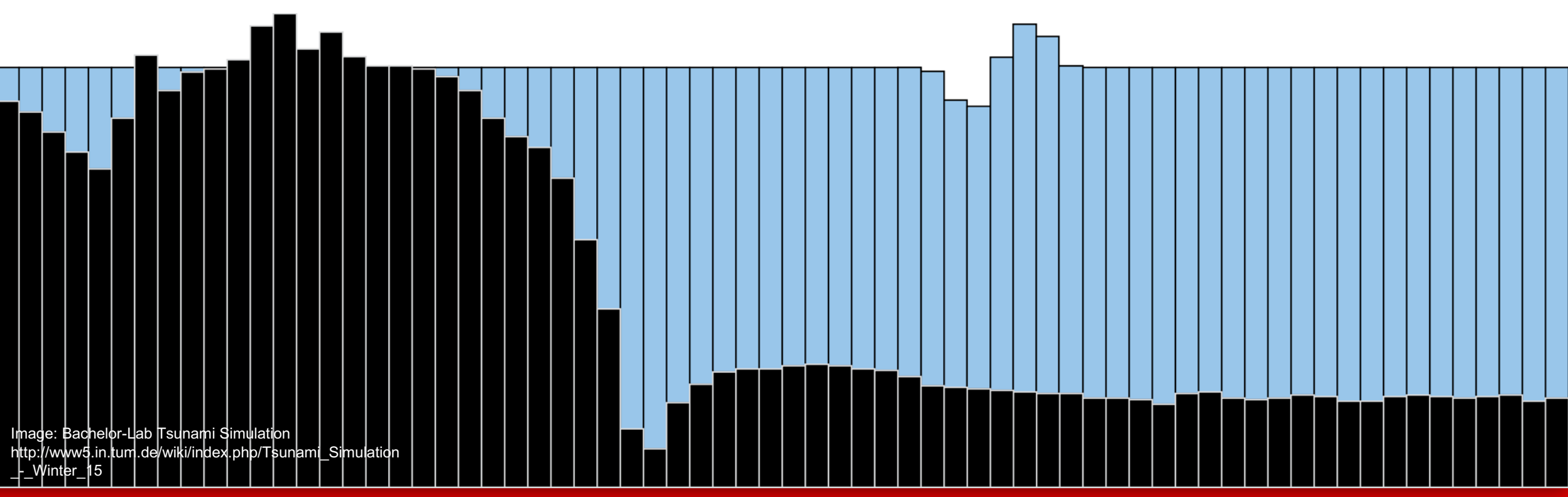









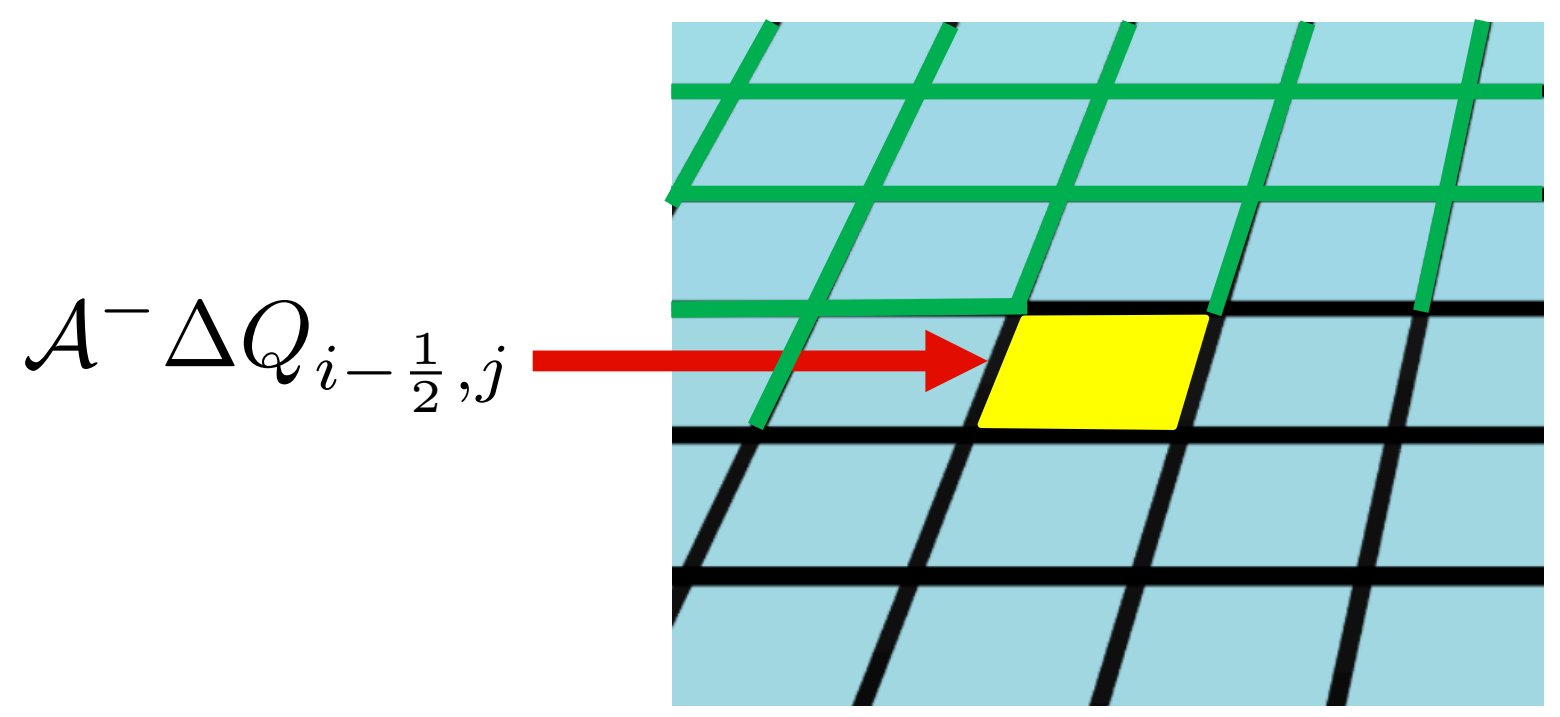




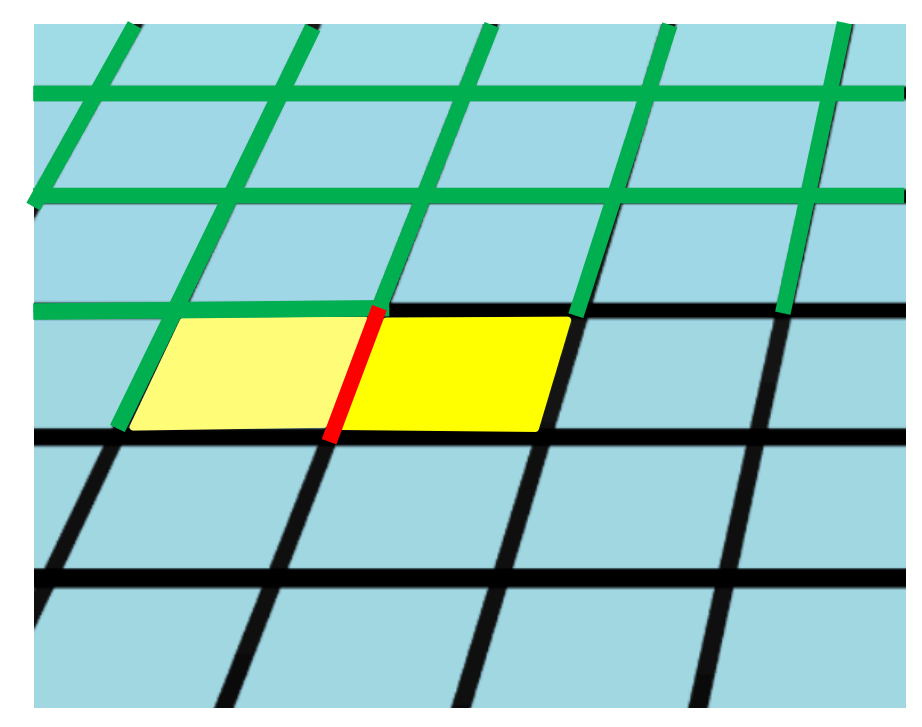




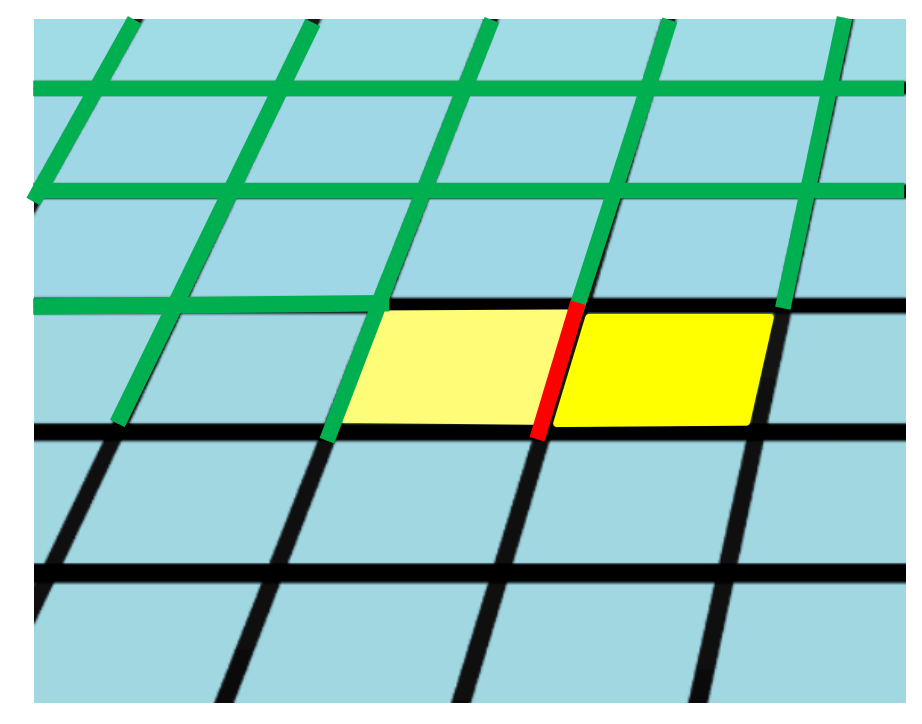




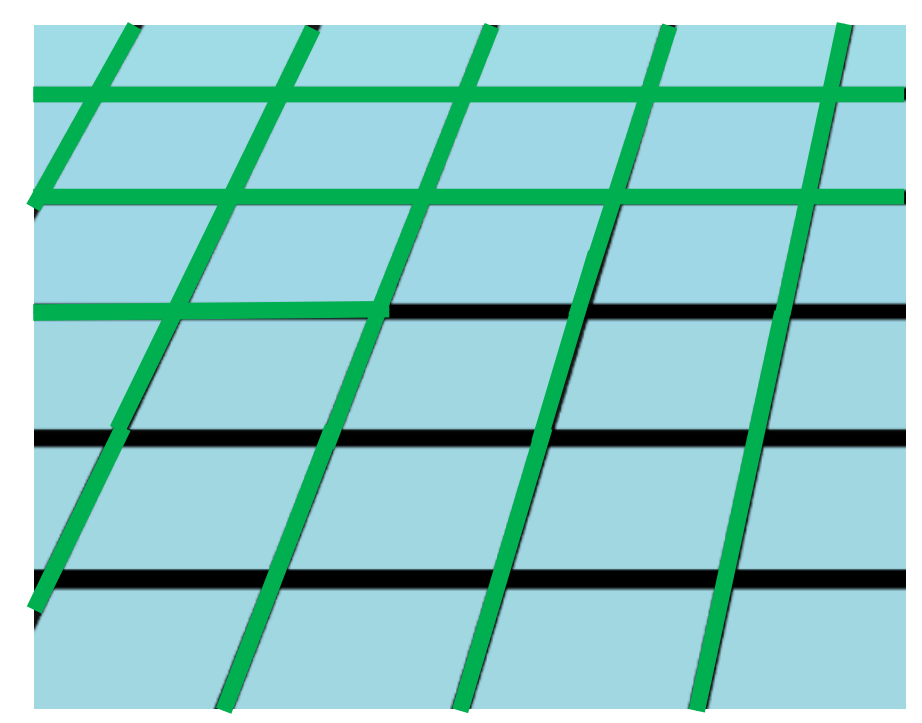









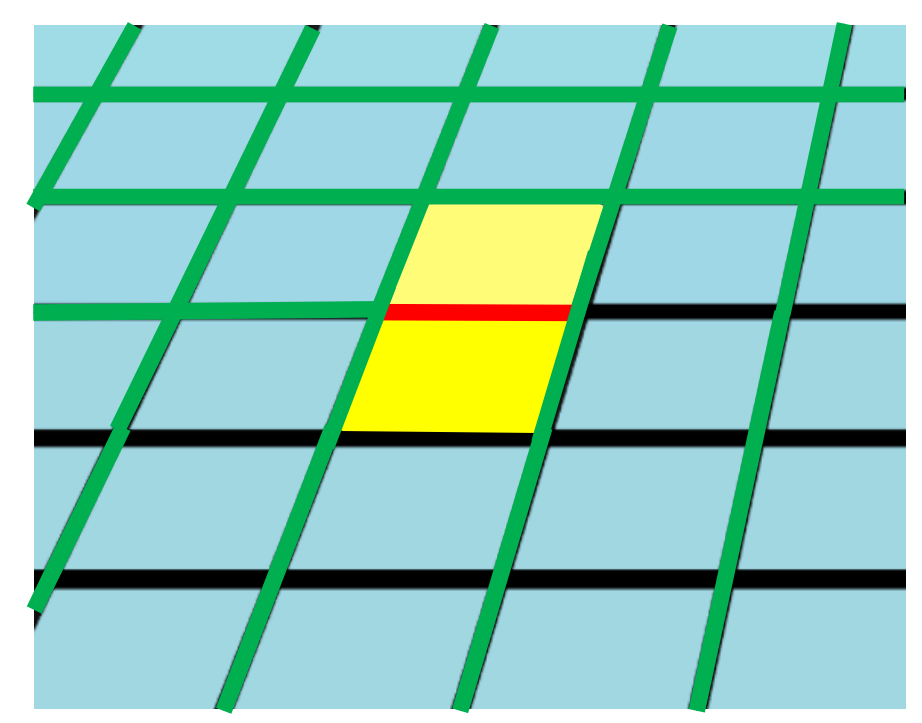




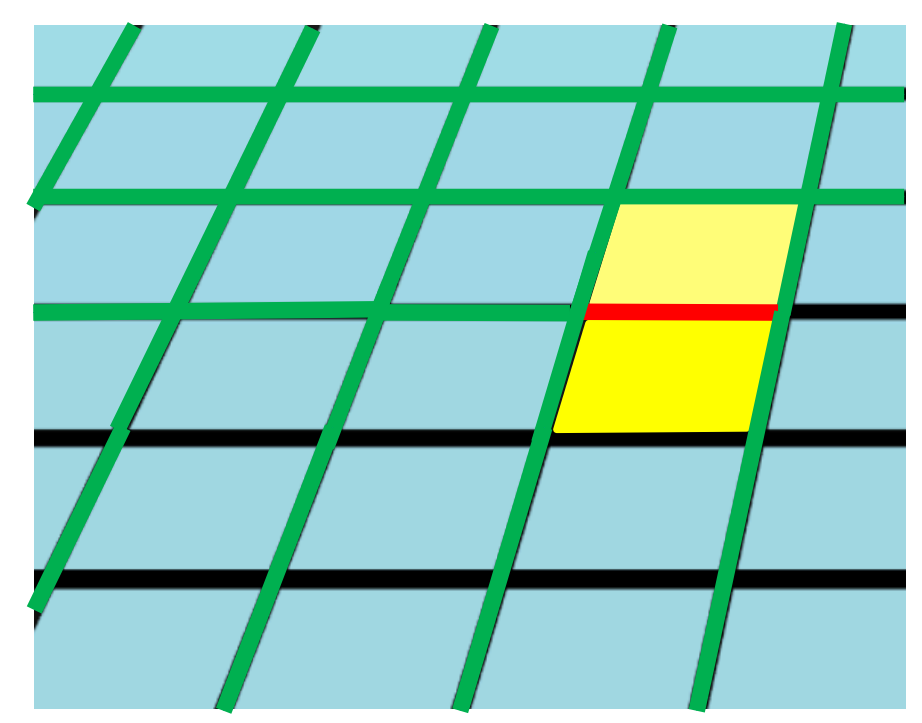




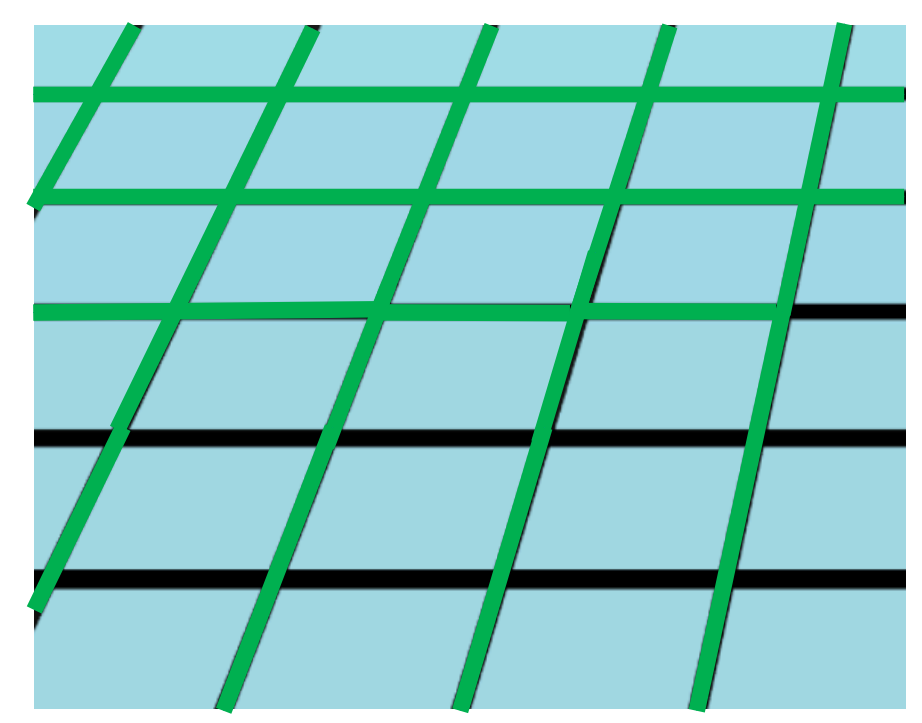


- Combination of "normal" CPU core and application-specific accelerators (through FPGA fabric)

- Realized through Custom Instructions $(\mathrm{Cl})$

- May be loaded at run time by application






\section{Acceleration of SWE-X10 using $i$-Core}

- Custom Instruction for computation of approximate solutions of Riemann Problems (f-Wave solver)

- Pipelined Accelerators (for operations used in solver):

-FP_MAC (3-5cy),

-FP_DIV (6 cy),

- FP_SQRT (5 cy),

-FP_UTIL (3 cy)

- Performs all 54 floating point operations as single $\mathrm{Cl}$

- Data-flow graph with 97 nodes/operations

- 5 accelerators used: 2x FP_MAC, 1x FP_DIV, 1x FP_SQRT and 1x FP_UTIL

- Configuration at application startup 


\section{Previous}

Current

Next

\section{Tile-Local Memory}


Previous

Current

Next 


\section{Adaptions in SWE-X10}



\section{Previous}

Current

$$
\text { Next }
$$

\section{Tile-Local Memory}




\section{Adaptions in SWE-X10}

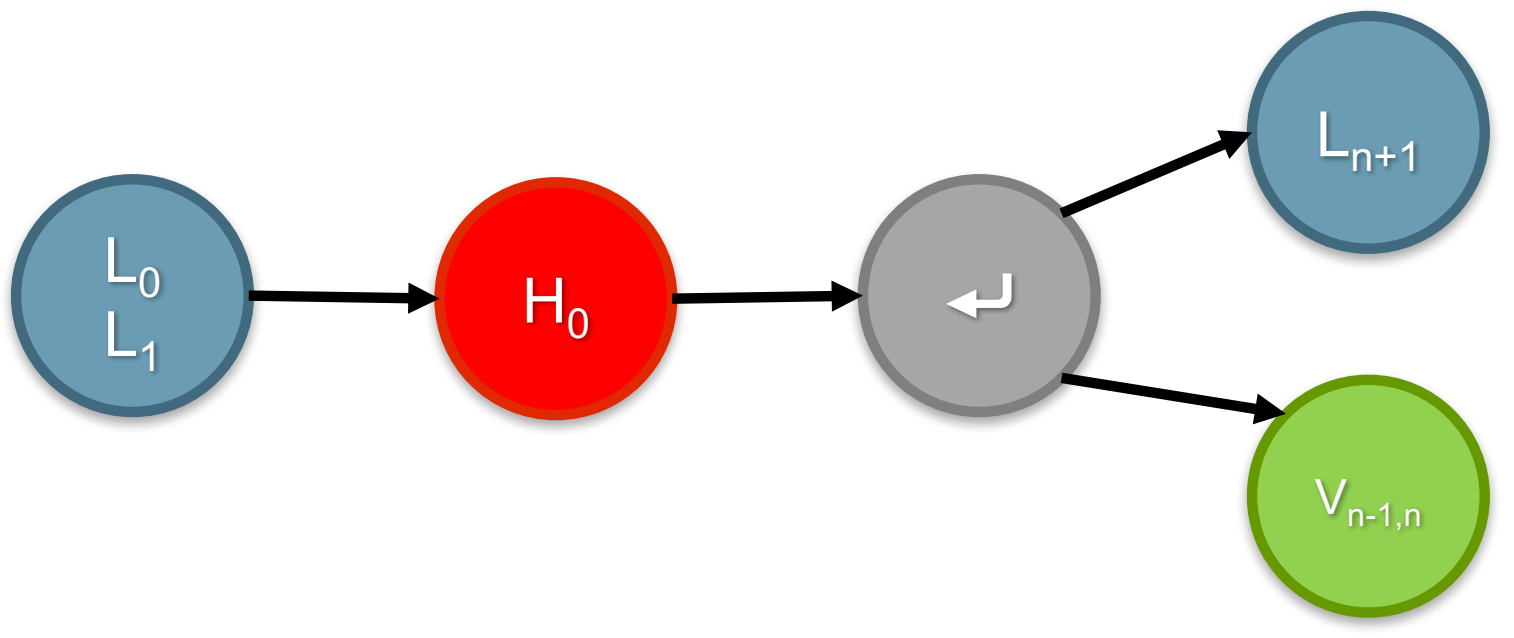

Previous

Current

Next

\section{Tile-Local Memory}




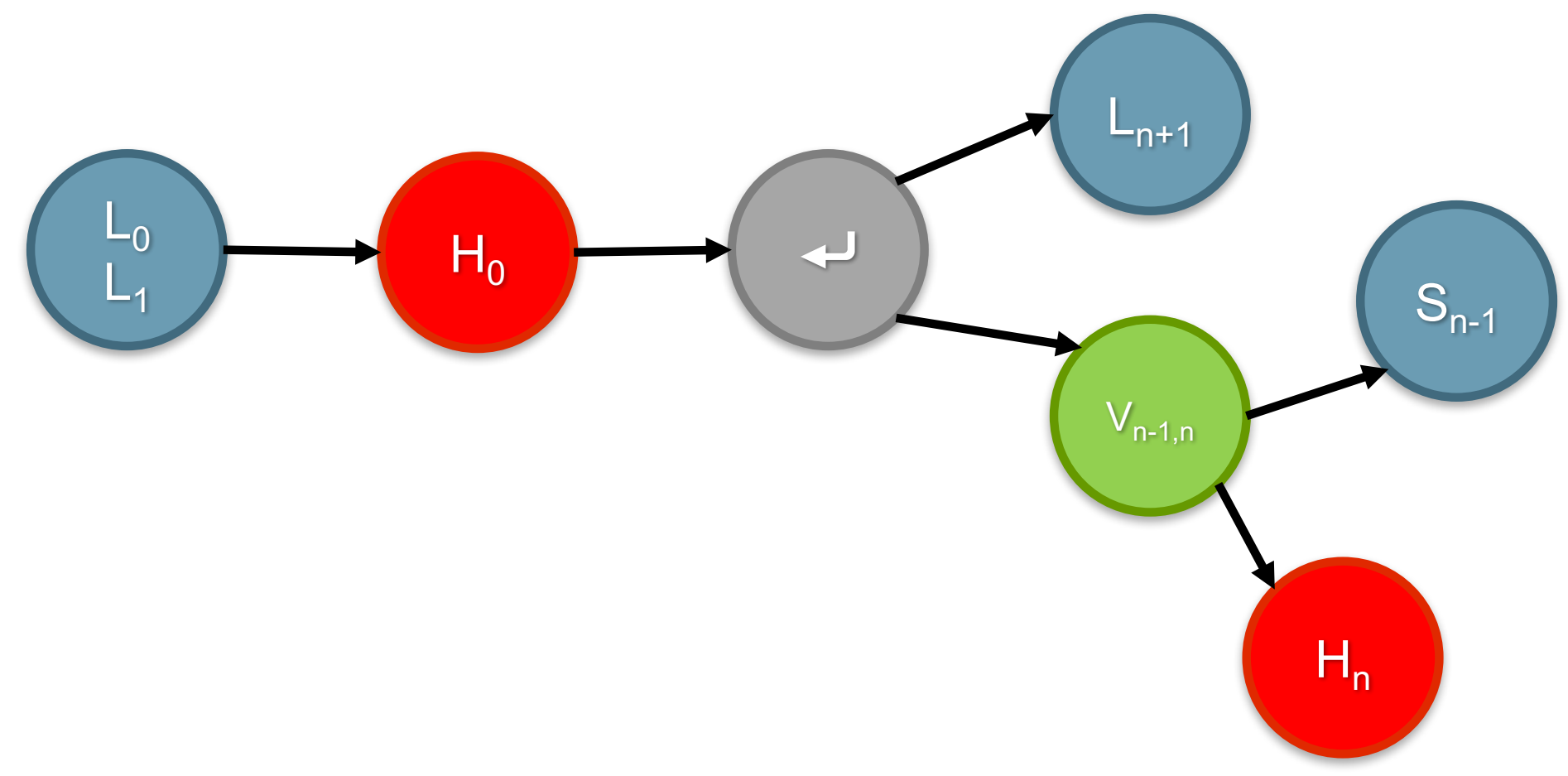

Previous

Current Next

\section{Tile-Local Memory}




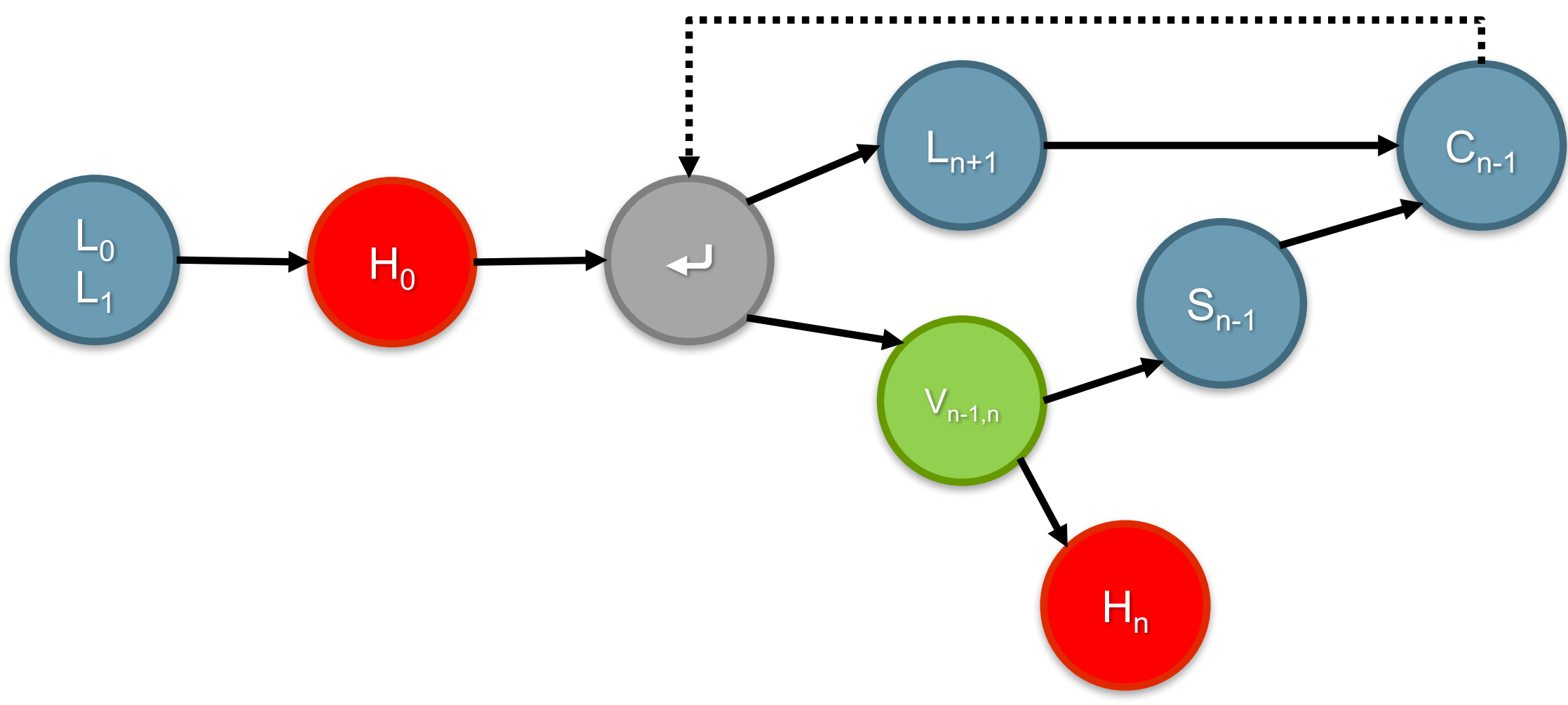

Previous

Current

Next

\section{Tile-Local Memory}




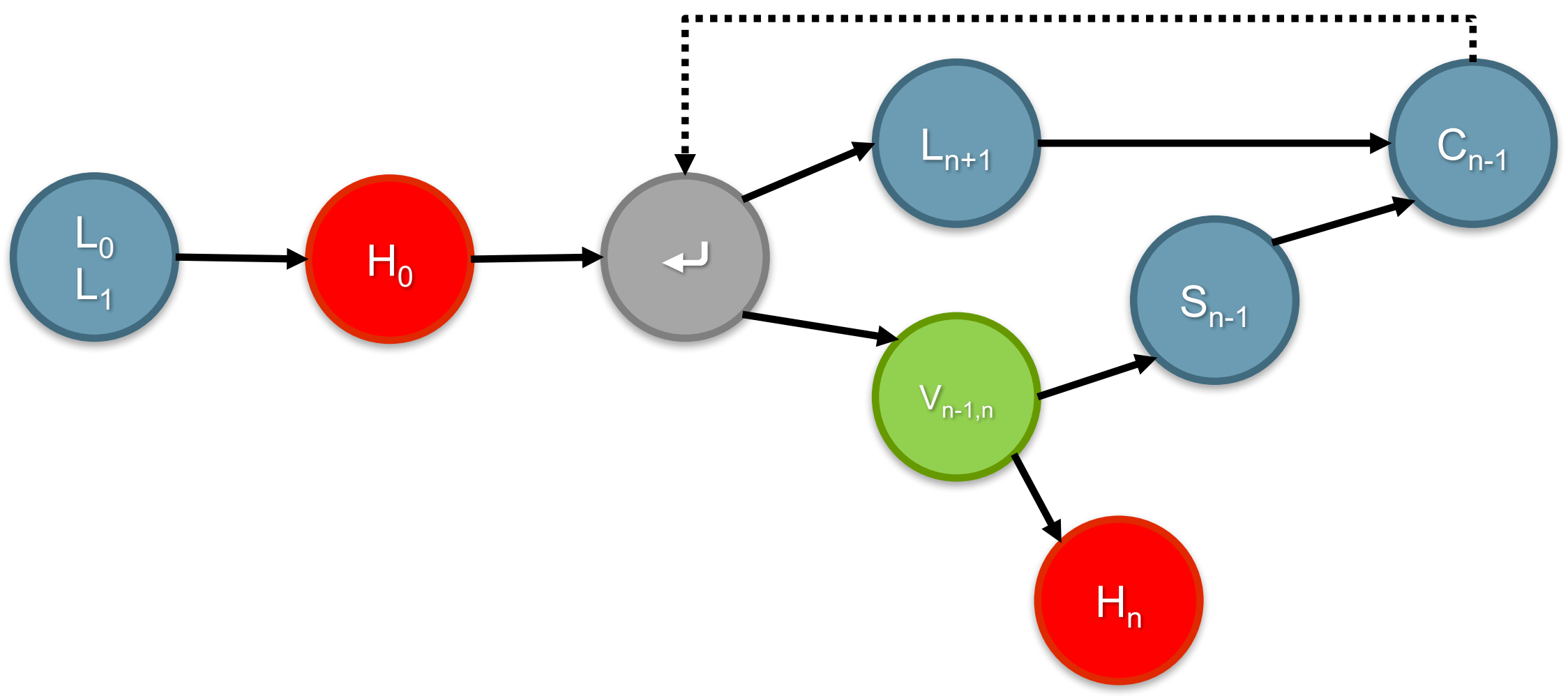

Previous Current Next

\section{Tile-Local Memory}




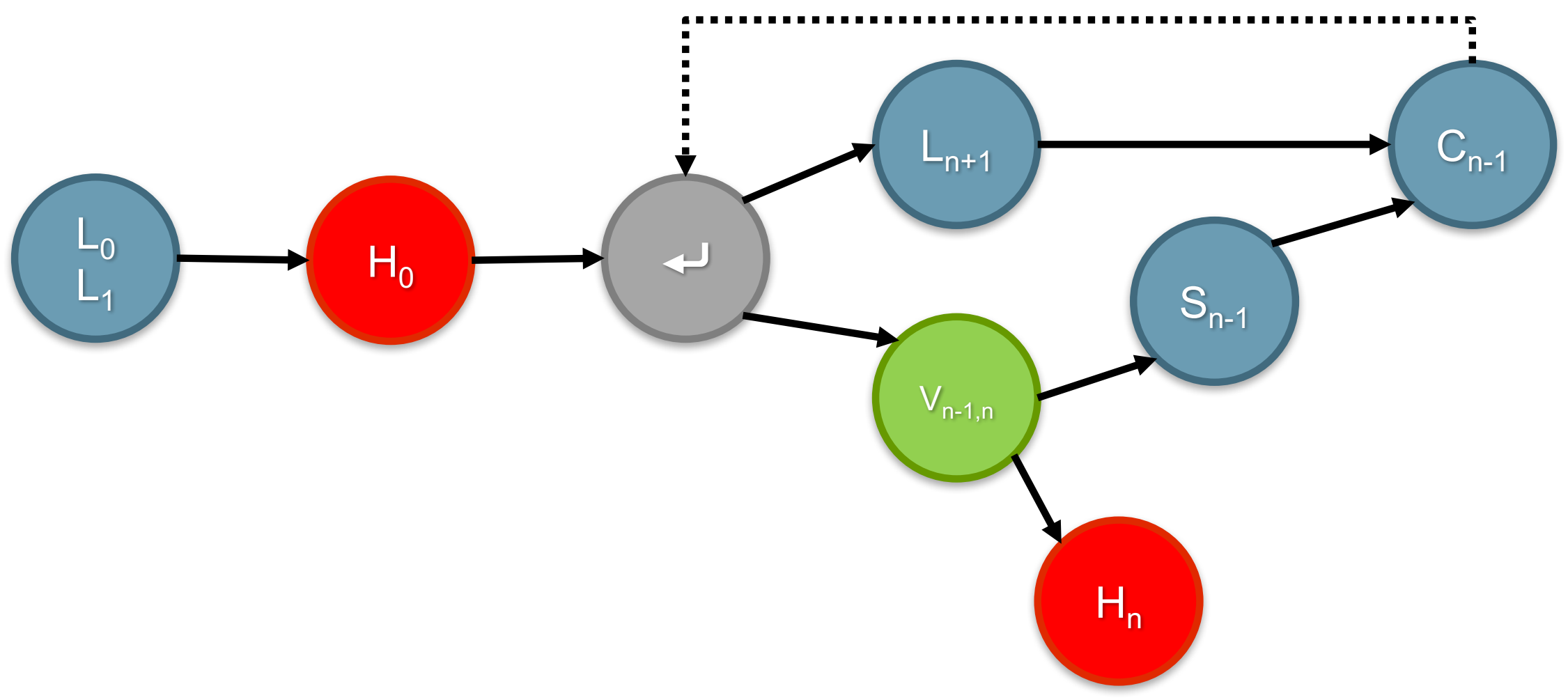

Previous

Current

Tile-Local Memory 




Previous Current Next

\section{Tile-Local Memory}




\section{Evaluation}

- Two Potential sources of performance gain:

- Tile-local memory

- i-Core

- Single iteration on one patch with $60 \times 60$ grid cells

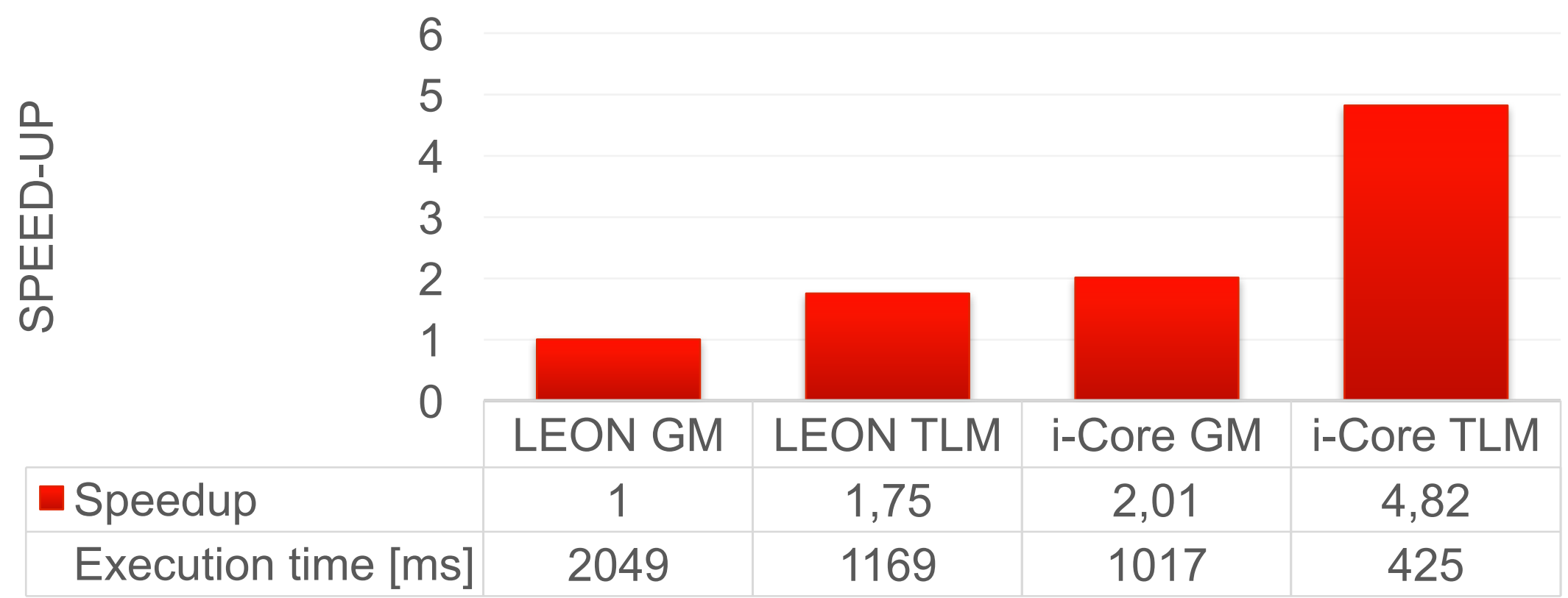




\section{Outlook}

- Model HLLE Riemann solver - enable coastal flooding

- Evaluate whole-system performance benefits

- Scale to and evaluate with larger hardware configuration (e.g. $4 \times 4$ tiles with $\sim 64$ cores multiple $i$-Cores)
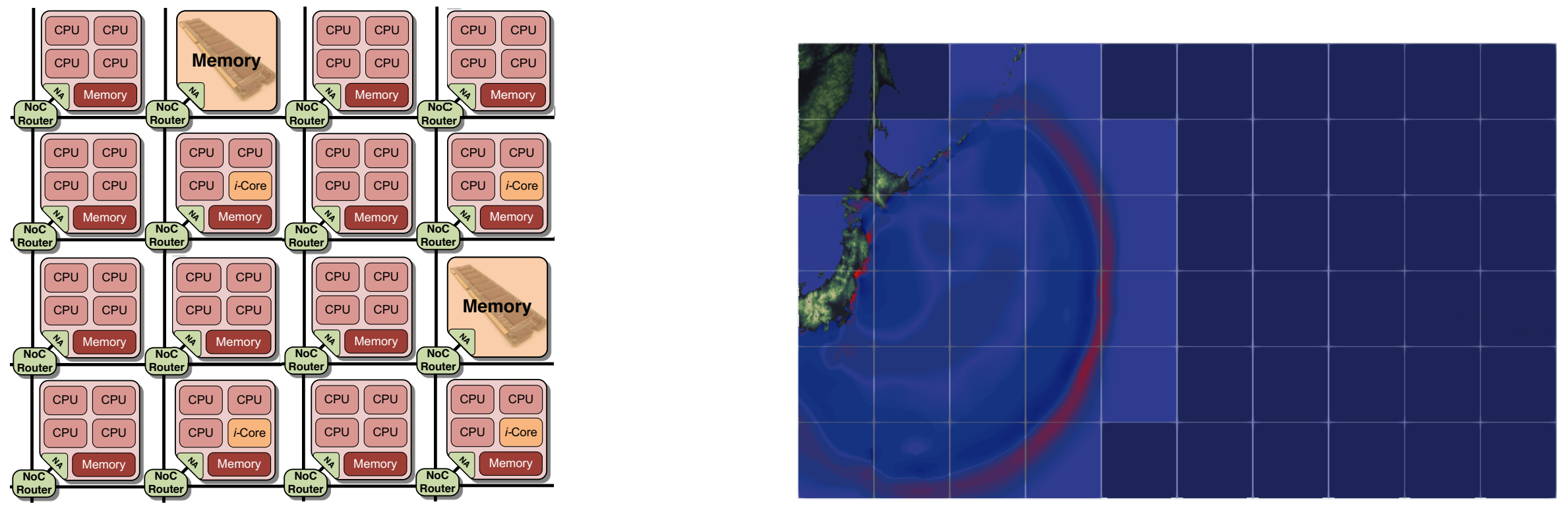


\section{Questions?}

\section{Acknowledgements}

This work was supported by the German Research Foundation (DFG) as part of the Transregional Collaborative Research Centre "Invasive Computing" (SFB/TR 89). 